\title{
ORIGEN ÉTNICO Y ANCESTRÍA GENÉTICA: ¿QUÉ LUGAR TIENEN EN LA PRÁCTICA MÉDICA EN ARGENTINA?
}

\author{
ETHNIC ORIGIN AND GENETIC ANCESTRY, WHAT PLACE DO THEY HAVE IN \\ MEDICAL PRACTICE IN ARGENTINA?
}

\author{
Anahí Ruderman ${ }^{1 *}$, Carolina Paschetta', Rolando González-José1 y Virginia Ramallo

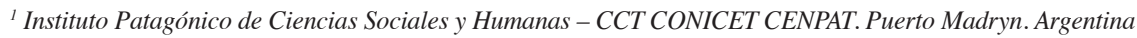

PALABRAS CLAVES: etnia; genómica poblacional; medicina de precisión

RESUMEN La investigación biomédica está contemplando cada vez más la ancestría genética de las poblaciones, con vistas a potenciales aplicaciones en la prevención sanitaria y en el tratamiento de dolencias población-específicas. Los objetivos fueron indagar sobre la formación en genética, universitaria y posuniversitaria, de médicos/as del país, y relacionarlo con la manera de abordar conceptos de genética médica poblacional en la práctica clínica. Se elaboró una encuesta semiestructurada dirigida a profesionales de Argentina. La encuesta se distribuyó en instituciones de médicos/ as de todas las provincias, tales como asociaciones, colegios, hospitales, foros, etc. Se obtuvieron 544 respuestas. El $78,5 \%$ de los encuestados recibió contenidos de genética en la carrera universitaria, siendo mayor este porcentaje entre profesionales jóvenes. El 98,5\% indaga sobre aspectos here- ditarios/genéticos relacionados con la enfermedad, mientras que el $64,5 \%$ consulta sobre el origen étnico. De este grupo, el 6,2\% se basa en los rasgos físicos externos (por ejemplo, su color de piel), y/o el apellido y/o el acento para asignarle un origen étnico. Quienes poseen formación de posgrado en genética, consultan sobre el origen poblacional del paciente en mayor medida que quienes no poseen esta formación. No existe un criterio unificado sobre cómo registrar la ancestría, el origen geográfico o la etnia en la práctica médica. Esto puede deberse a que dichas variables no son especialmente destacadas, desde la formación académica misma. Reforzar contenidos de genética médica poblacional puede servir para valorizar este aspecto de la casuística de las enfermedades complejas. Rev Arg Antrop Biol 22(2), 2020. doi: $10.24215 / 18536387 \mathrm{e} 020$

\section{KEYWORDS ethnicity; population genomics; precision medicine}

ABSTRACT Increasingly, biomedical research is looking at the genetic ancestry of populations with a view to its potential applications in health prevention, as well as in the treatment of population-specific diseases. The objective of this work was to investigate the training in genetics at graduate and postgraduate levels of physicians in the country, and relate it to the way of approaching concepts of population medical genetics in clinical practice. A semi-structured survey was made for professionals from Argentina. The survey was distributed to institutions of physicians of all the provinces, such as associations, schools, hospitals, forums, etc. 544 answers were gathered. $78.5 \%$ of respondents received genetics-related content in their university syllabus, this percentage being higher among young professionals. $98.5 \%$

Algunas enfermedades presentan más incidencia en una población que en otra de distinto origen. Un ejemplo de esto es el cáncer de mama: las tasas de incidencia son más altas en mujeres de ancestría europea con respecto a mujeres de ancestría asiática. Son diversos los factores que influyen en este mapa epidemiológico; uno de ellos es la variabilidad en la frecuencia de variantes genéticas de predisposición para enfermedades (Bains et al., 2013; Baye, Wilke y Olivier, 2009). En los últimos años, se ha comenzado a prestar más atención a la estructura inquire about hereditary/genetic aspects related to the disease, while $64.5 \%$ inquire about the ethnic origin. Of this group, $6.2 \%$ rely on external physical features (for example, skin color), and/or surname and/or accent to assign an ethnic origin. Those who have postgraduate training in genetics ask about the population origin of the patient to a greater extent than those who do not have this training. There is no unified criterion on how to register ancestry, geographic origin or ethnicity in medical practice. This may be due to a low relative importance attributed to these variables, from the academic training itself. Strengthening contents of population medical genetics can serve to enhance this aspect of the causes of complex diseases. Rev Arg Antrop Biol 22(2), 2020. doi:10.24215/18536387e 020

genética fina de las poblaciones y a considerarla un factor importante en el estudio de las enfer-

Financiamiento: Agencia Nacional de Promoción Científica y Tecnológica, PICT 2015-3206 Tipo A; Consejo Nacional de Investigaciones Científicas y Técnicas, PIP 2015-2017 GI.

*Correspondencia a: Anahí Ruderman. CCT CONICET-CENPAT. Bv. Almirante Brown 2915. U9120ACD Puerto Madryn. Argentina. E-mail: ruderman@cenpat-conicet.gob.ar.

Recibido 26 Abril 2019; aceptado 22 Octubre 2019

doi: $10.24215 / 18536387 \mathrm{e} 020$ 
medades complejas: la investigación en salud está contemplando cada vez más la ancestría genética de las poblaciones, tanto a nivel local como regional.

Este cuerpo de conocimiento ha comenzado a trasladarse a la práctica clínica. La medicina de precisión, un paradigma emergente en el campo del cuidado de la salud, se basa en la caracterización de los factores hereditarios (variantes genéticas de susceptibilidad) así como de los factores de riesgo adquiridos (historia de vida, el género, nutrición durante el crecimiento, consumo de tabaco y otras drogas, pautas culturales, etc.) para establecer un modelo del riesgo de una persona con respecto a un posible estado de salud/enfermedad. Sin embargo, la precisión de la medicina de precisión está construida, hasta ahora, sobre la base de poblaciones principalmente de origen europeo. Existe un enorme desbalance entre el número de estudios genómicos de base poblacional entre Europa y el resto de los continentes (Landry y Rehm, 2018; Landry, Ali, Williams, Rehm y Bonham, 2018).

En algunos países, como en Estados Unidos, los productos de ancestría y salud están ganando popularidad entre el público general (Ramos y Weissman, 2018) y la secuenciación con fines clínicos es utilizada de manera rutinaria para el diagnóstico, el pronóstico y el tratamiento. Sin embargo, en países con menos recursos, este servicio no es masivo debido a su elevado costo.

Para conocer el origen poblacional de un paciente, es usual en el ámbito clínico el registro de la etnia auto-percibida, o bien el registro de la etnia que el profesional considera de acuerdo a su propio criterio. Sin embargo, la ancestría o etnia auto-reportada es informativa de la percepción de la persona sobre sí, es el resultado del proceso del individuo en relación con su cultura y puede variar a lo largo de la vida en función de los sistemas de relaciones y de variables políticas. Esta autodefinición puede ser particularmente compleja en poblaciones que han experimentado recientes eventos de mestizaje. Por ejemplo, en un estudio donde se comparó la ancestría genética y la auto-percibida en poblaciones latinoamericanas, se encontró una correlación significativa entre estas dos aproximaciones, pero también se observó que determinados rasgos corporales sesgan la auto-percepción, resultando en una gran disparidad con la estimación genética (Ruiz-Linares et al., 2014). Por lo tanto, la identidad étnica puede no ser necesariamente un buen proxy de la compleja historia genética de un individuo (Mersha y Abebe, 2015), la cual es muy variable entre personas y poblaciones y sólo puede ser capturada a través de datos genómicos de gran escala.

El registro de la etnia auto-percibida puede presentar dificultades, particularmente cuando es aplicado por las instituciones de salud de países con población mestiza (Travassos y Williams, 2004). Un ejemplo es el Censo Nacional de Estados Unidos del año 2000, donde podía incluirse más de una respuesta sobre la etnicidad auto-percibida. En este censo, un gran número de personas reportó ser de más de una categoría étnica, seleccionando en algunos casos "blanco" y "negro" por igual. Por otro lado, las categorías étnicas incluidas en las planillas de los hospitales o clínicas generalmente son insuficientes e imprecisas con respecto a la variabilidad de la población. Los desafíos incluyen las complejidades del mestizaje ancestral y las diferentes interpretaciones de las categorías étnicas y de ancestría (Popejoy et al., 2018).

En este contexto, surge el interés de saber cómo los médicos y médicas de Argentina, especialistas en enfermedades complejas como la obesidad, la diabetes y el síndrome metabólico, construyen y utilizan nociones relacionadas con la ancestría y el origen poblacional. Los objetivos son conocer cómo es la formación en genética a nivel universitario y pos universitario en las carreras de medicina del país, y relacionarlo con la manera de abordar conceptos de genética médica poblacional en la práctica clínica diaria.

\section{MATERIAL Y MÉTODOS}

Se elaboró una encuesta semiestructurada dirigida a médicos y médicas que cursaron sus estudios formales en Argentina y que ejercen la profesión en el país. Durante el proceso de elaboración de la herramienta de recolección de datos, se llevaron a cabo reuniones con profesionales en medicina, con el fin de validar las preguntas individuales y el instrumento en su conjunto. Se indagó sobre la pertinencia de las interrogaciones desarrolladas así como de las opciones de respuesta incluidas, de manera que la encuesta fuera de validez, confiabilidad y uti- 
lidad a los fines de nuestra investigación, contemplando un lenguaje más específico y cercano al utilizado por los/as profesionales de la salud.

La encuesta incluyó preguntas sobre formación universitaria y pos universitaria y la especialidad médica del/la encuestado/a (universidad donde obtuvo su título, formación en relación a genética, cursos de posgrado de genética). También se indagó sobre aspectos de la práctica clínica en cuanto a la aplicación de conceptos hereditarios/genéticos y de ancestría/ etnia y sobre las formas de utilizar dicha información durante la relación con el/la paciente.

Una vez obtenida la versión final de la encuesta, se contrató una página web especializada en encuestas online, que permitió recopilar las respuestas de manera rápida, organizada y segura.

Para su comunicación y distribución, se estableció contacto con instituciones de todas las provincias del país, tales como asociaciones profesionales, colegios de médicos/as, hospitales, foros, etc. Aquellas instituciones que a través de sus respectivos órganos directivos dieron su aprobación, distribuyeron la encuesta entre sus miembros vía correo electrónico y/o páginas web.

\section{RESULTADOS}

Se obtuvieron un total de 544 respuestas de profesionales de todo el país cuyas especialidades variaron entre: Clínica médica, Psiquiatría, Oftalmología, Traumatología, Hematología, Ginecología y Obstetricia, Neonatología y pediatría, Genética médica, Medicina legal o del trabajo, Infectología, Diabetología y Endocrinología, entre otras. Entre las personas encuestadas, 113 trabajaban en el sector público de la salud, 150 en el sector privado y 268 en el ámbito público tanto como privado. En la Figura 1 se pueden observar las frecuencias de respuestas por universidad.

Con respecto al año de egreso de la carrera universitaria, las respuestas variaron entre 1963 y 2017. Dividiendo la muestra en bloques de 10 años, la cantidad de respuestas estuvo distribuida homogéneamente excepto por el primer bloque (1963-1973) donde la cantidad de respuestas fue varias veces menor al resto.

Se dividió la muestra en tres categorías de acuerdo a las especialidades. La categoría 1 incluyó aquellas que están directamente vinculadas a las dolencias complejas de nuestro interés: Nutrición, Diabetología, Endocrinología, Clínica, Medicina familiar, Genética médica. En la categoría 2 se incluyeron las especialidades que están indirectamente vinculadas a las dolencias complejas de nuestro interés: Cardiología, Oncología, Ginecología, Hematología, Pediatría. Por último, la categoría 3 contiene médicos/as cuyas especialidades guardan una relación muy indirecta con las dolencias de interés: Traumatología, Oftalmología, Dermatología, Cirugía, Terapia intensiva, Psiquiatría, Reumatología, Infectología, ORL, Nefrología, Medicina legal/del trabajo, Anatomía patológica. Las frecuencias y los porcentajes de cada categoría se encuentran en la Tabla 1.

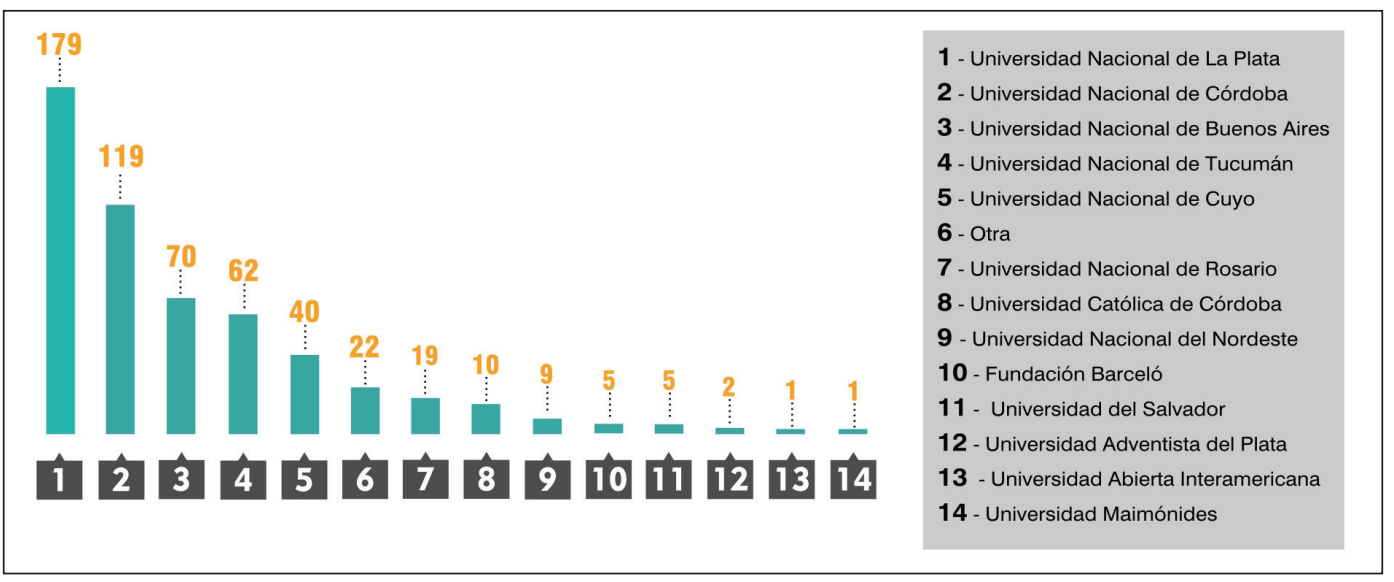

Fig. 1. Frecuencia de respuestas según la universidad de procedencia. 
TABLA 1. Frecuencia y porcentaje de respuestas según categoría de especialidad

\begin{tabular}{ccc}
\hline $\begin{array}{c}\text { Categoría de } \\
\text { especialidad }\end{array}$ & Frecuencia & $\%$ \\
\hline 1 & 200 & 36.7 \\
2 & 132 & 24.3 \\
3 & 212 & 39.0 \\
\hline Total & 544 & 100 \\
\hline
\end{tabular}

\section{Sobre la formación en genética en la carrera}

Sobre este tópico, 427 personas $(78,5 \%)$ respondieron que habían recibido contenidos de genética, contra 117 (21,5\%) que respondieron lo contrario. De aquellas cuya respuesta fue positiva, 308 se refirieron a Genética médica, 74 a Genética del desarrollo, 21 a contenidos de Genética de poblaciones, 90 a Genética molecular, 61 a contenidos en Citogenética, 60 a Mutagénesis, 2 a Genética cuantitativa y 14 a otras áreas. Un total de 381 médicos/as $(71,1 \%)$ no realizaron cursos, escuelas o seminarios sobre genética por fuera de la carrera universitaria, contra 155 (28,9\%) encuestados/as que sí adquirieron este tipo de formación.

\section{Sobre los aspectos hereditarios y el origen étnico del/la paciente}

Casi la totalidad de la muestra $(98,5 \%)$ indagaba sobre aspectos hereditarios/genéticos relacionados con la enfermedad o dolencia del/la paciente, por ejemplo, preguntando por antecedentes familiares en padres y abuelos.

Por otro lado, el $64,5 \%$ de los/as encuestados/as indagaba de alguna manera sobre el origen poblacional o étnico. De este grupo, el $70,3 \%$ preguntaba al paciente por su origen geográfico/étnico/nacionalidad auto-reportado, o por el de sus padres y/o abuelos. El 6,2\% se basa en los rasgos físicos externos (por ejemplo, su color de piel) y/o en el apellido y/o en el acento del/de la paciente para asignarle un origen geográfico/étnico/nacionalidad. Por último, el $23 \%$ restante utilizaba las dos estrategias: consulta por el origen auto-percibido y también se basaba en su propio criterio para asignarle un origen poblacional (según rasgos físicos externos, acento y/o el apellido). Otras respuestas diferentes a las opciones incluidas en la encuesta fueron: "le asigno un origen poblacional/étnico por saludar en el dialecto del paciente", "le pregunto si es adoptado", entre otras.

Un total de 14 encuestados/as no utilizaban la información respecto a antecedentes familiares u origen poblacional/étnico. Por otro lado, 288 personas utilizaban la información tanto de antecedentes familiares como sobre el origen poblacional/étnico, 195 médicos/as solo utilizaban la información sobre antecedentes familiares de la enfermedad y 12 personas utilizaban la información sobre el origen poblacional/étnico, pero no sobre antecedentes familiares.

Una mínima proporción (10 personas) consideraba que sus pacientes reciben de manera negativa las preguntas acerca de herencia o ancestría genética, mientras que 165 consideraban que lo toman de manera neutral y 298 que lo reciben de manera positiva.

La Tabla 2 describe en qué etapas del proceso se utiliza la información recabada.

Se analizó la relación entre el año de egreso de la carrera y la formación en genética. Los/ as profesionales más jóvenes (años de egreso más cercanos al presente), manifestaron en mayor porcentaje haber tenido formación académica en genética. Sin embargo, contrario a lo anterior, cuando se analizó si tiene en cuenta el origen poblacional/étnico durante su práctica clínica, el porcentaje de afirmaciones fue menor entre profesionales más jóvenes (Tabla 3), aunque la diferencia entre los grupos no fue significativa $(p=0,4)$

TABLA 2. Etapas donde es utilizada la información sobre antecedentes familiares y origen poblacional

\begin{tabular}{cc}
\hline Etapa & Frecuencia \\
\hline En prevención & 237 \\
Al realizar un diagnóstico & 381 \\
Al asignar un tratamiento & 79 \\
Al recetar fármacos & 31 \\
Otra & 47 \\
\hline
\end{tabular}


TABLA 3. “ ¿Indaga sobre origen poblacional o étnico?” “ “Recibió contenidos de genética en la carrera?” de acuerdo al año de egreso

\begin{tabular}{|c|c|c|c|c|}
\hline Año de egreso & $\begin{array}{l}\text { Formación académica } \\
\text { en genética }(\%)\end{array}$ & $\begin{array}{l}\text { Indaga sobre origen } \\
\text { poblacional }\end{array}$ & $\mathrm{N}$ & $\%$ \\
\hline \multirow{3}{*}{$1963-1973$} & \multirow{3}{*}{79,3} & No & 8 & 29,6 \\
\hline & & $\mathrm{Si}$ & 19 & 70,4 \\
\hline & & Total & 27 & 100 \\
\hline \multirow{3}{*}{ 1974-1984 } & \multirow{3}{*}{72,6} & No & 31 & 30,7 \\
\hline & & $\mathrm{Si}$ & 70 & 69,3 \\
\hline & & Total & 101 & 100 \\
\hline \multirow{3}{*}{ 1985-1995 } & \multirow{3}{*}{76,2} & No & 36 & 34,3 \\
\hline & & $\mathrm{Si}$ & 69 & 65,7 \\
\hline & & Total & 105 & 100 \\
\hline \multirow{3}{*}{$1996-2006$} & \multirow{3}{*}{80,0} & No & 66 & 37,7 \\
\hline & & $\mathrm{Si}$ & 109 & 62,3 \\
\hline & & Total & 175 & 100 \\
\hline \multirow{3}{*}{$2007-2017$} & \multirow{3}{*}{83,0} & No & 47 & 38,8 \\
\hline & & $\mathrm{Si}$ & 74 & 61,2 \\
\hline & & Total & 121 & 100 \\
\hline \multirow{3}{*}{ Total } & \multirow{3}{*}{78,2} & No & 188 & 35,5 \\
\hline & & $\mathrm{Si}$ & 341 & 64,5 \\
\hline & & Total & 529 & 100 \\
\hline
\end{tabular}

Al analizar por especialidad, el $66,7 \%$ de los/las profesionales que pertenecen a la categoría 1 consideraba que el origen poblacional/étnico de sus pacientes es una variable que debe ser tenida en cuenta, al igual que el $60 \%$ de los profesionales de la categoría 2 y el $63,1 \%$ de los profesionales de la categoría 3 . Las diferencias entre estos grupos no fueron significativas.

Se indagó si la formación a través de cursos, escuelas o seminarios de genética influye en la práctica del profesional, al momento de tener en cuenta o no el origen poblacional de sus pacientes (Tabla 4). Entre quienes asistieron a algún tipo de formación en genética por fuera de la educación recibida durante la carrera, el 75,8\% manifestó tenerlo en cuenta. Por otro lado, de aquellos/as que no asistieron a alguna actividad de formación en genética, el 59,8\% indaga sobre el origen del paciente. Cuando se aplicó una prueba de Chi-cuadrado para comparar estos porcentajes, se observó una diferencia estadísticamente significativa $(p=0,000)$.

\section{DISCUSIÓN}

\section{La etnia auto-reportada en el contexto de la historia clínica}

La historia clínica es el documento en el que cada profesional registra una serie de variables de relevancia que luego serán consideradas a la hora de realizar un diagnóstico, aplicar un tratamiento, recetar fármacos, etc. En Argentina, si bien es un documento obligatorio, no existe una manera única de completarlo y cada institución/ profesional la aborda según sus criterios. Menos aún existe una forma unificada de registrar la ancestría, el origen geográfico o la etnia. Por lo tanto, es esperable que algunas personas tengan en cuenta estos aspectos mientras que otras no. 
TABLA 4. Porcentaje de profesionales que consultan por origen poblacional/étnico, según su formación de posgrado en genética

\begin{tabular}{cccc}
\hline $\begin{array}{c}\text { Asistieron a curso, escuela, seminario de } \\
\text { genética }\end{array}$ & $\begin{array}{c}\text { Pregunta por origen poblacional } \\
\text { o étnico }\end{array}$ & N & \% del total \\
\hline \multirow{2}{*}{ No } & No & 151 & 40,2 \\
& Sí & 225 & 59,8 \\
\hline \multirow{2}{*}{ Sí } & No & 37 & 24,2 \\
& Sí & 116 & 75,8 \\
\hline
\end{tabular}

Tal fue el patrón que observamos en el presente estudio.

El $64,5 \%$ de encuestados/as indaga sobre el origen poblacional o étnico de sus pacientes. Coexisten múltiples formas de consignar esta variable, siendo más frecuente $(70,3 \%)$ la consulta al/a la paciente sobre su identidad auto-reportada, o el de sus padres y/o abuelos. Como ya se mencionó anteriormente, las identidades auto-percibidas no necesariamente tienen un correlato con las variables genéticas y las nociones históricas, sociales y culturales toman un rol fundamental. La imposición durante siglos de un sistema de clasificación humano en razas, ejercida por grupos dominantes de países centrales con fines económicos y políticos, ha sido una fuerza generadora de estigmatización, inequidades y segregación, entre otros fenómenos socialmente nocivos. Las consecuencias negativas persisten hasta el presente, por lo que es posible imaginar que algunas personas no se sientan plenamente libres al tener que responder sobre su origen étnico en el marco de una consulta con un/una profesional de la salud. Esto puede conducir hacia respuestas sesgadas acordes a lo valorado positivamente, en la sociedad que se integra, en un momento histórico y un lugar determinado.

Cabe resaltar el gran porcentaje de médicos/ as que registran de esta forma la identidad del paciente, en comparación con quienes la consignan en forma directa en función de criterios personales $(6,2 \%)$. Estos últimos se basan en su juicio y apreciación sobre los rasgos físicos externos (por ejemplo, el color de piel), y/o en el apellido y/o en el acento. En este sentido, se ha observado que la clasificación visual del color de la piel puede ser interpretada de manera diferente por pacientes, trabajadores de la salud y médicos/as de familia (Parra et al., 2003). Esta variable fenotípi- ca no necesariamente está vinculada de manera unidireccional con el origen o la etnia, sino que expresa relaciones e interacciones gen-ambiente más complejas, sobre todo en poblaciones mestizas. Asímismo, aquellas instituciones que utilizan formularios estandarizados incluyen categorías de clasificación que no capturan la variabilidad de la población en su totalidad. Más aún, ciertas categorías pueden tener un significado para una persona y uno muy distinto para otra. Se suelen simplificar como "blanca" o "caucásica", "asiática", "africana" o "latina", sin lograr una buena aproximación a la diversidad biológica humana ni contemplar individuos con ascendencia de más de un origen y en distintas proporciones (Mersha y Abebe, 2015). El aspecto genético está influenciado por variables diferentes de la etnicidad o identidad auto-percibida. Un posible camino es generar un modelo de pregunta, que permita registrar la etnia o ancestría auto-percibida de manera uniforme entre las distintas instituciones. Para ello, deberán trabajar en conjunto médicos/ as y científicos/as aportando a la discusión desde sus respectivos campos de estudio. Entre los desafíos de este proceso se destaca cuáles categorías incluir, considerando el extenso mestizaje de la población argentina. También cabe preguntar si es adecuado generar un único modelo de pregunta, cuando dentro del país es posible que las mismas categorías sean interpretadas de manera regionalmente diferente, según el contexto y su historia. La aplicación de este modelo de cuestionario podría estar precedida por una capacitación, de manera que de surgir dudas por parte de pacientes respecto al cuestionario, éstas puedan ser saldadas de manera consistente por profesionales de la salud.

En otro plano, una solución a futuro para cuantificar la ancestría en el ámbito clínico es el 
desarrollo de un chip de ancestría de bajo costo específico para la población argentina. Un paso necesario es generar una base de datos robusta y amplia, que permita caracterizar la variabilidad genética de nuestra población.

\section{La formación académica}

Al comparar por universidad de procedencia, no hubo diferencias entre los encuestados en la manera de considerar y utilizar el origen étnico del paciente en su práctica médica (Fig. 2 ). En este punto, fue más influyente la formación de posgrado y se analizará más adelante.

Las tres universidades más representadas en la muestra fueron: Universidad Nacional de La Plata, Universidad Nacional de Córdoba y Universidad de Buenos Aires.

En la actualidad, la carrera de Medicina de la Universidad Nacional de La Plata cuenta en su programa con la materia Genética (de modalidad optativa). En ella están incluidos temas como: "Construcción de bibliotecas genómicas", "Enfermedades con herencia compleja: diabetes mellitus, retinitis pigmentaria digénica, enfermedades de las arterias coronarias", "Mapeo de alta resolución" "Proyecto del Genoma Humano", "Estudios de las influencias genéticas y ambientales en las enfermedades complejas", "Aplicaciones del mapeo de genes humanos", "Mapeo de genes humanos mediante análisis de ligamiento", "Microarrays" y "Terapia génica".

El plan de estudios actual de la carrera de
Medicina de la Universidad Nacional de Córdoba no contiene la materia Genética. La materia Bioquímica y Biología molecular del primer año incluye, en una de sus unidades, pocos contenidos de genética tales como "Técnicas de microarray e interferencia por ARN. Terapia génica". En cuanto a la carrera de Medicina de la Universidad de Buenos Aires, en su primer año cuenta con la materia Histología, Biología celular, Embriología y Genética.

El 78,5\% de los encuestados aseguró haber recibido formación en genética durante la carrera universitaria. Al evaluar por edad, se observó un mayor porcentaje de respuestas afirmativas en este campo entre los/las profesionales más jóvenes. Llamativamente, también son quienes menos tienen en cuenta el origen poblacional/ étnico durante su práctica clínica. Si bien los programas más actuales de las carreras de Medicina estarían incorporando mayor cantidad de contenidos en genética médica, en relación a planes de estudio anteriores, no necesariamente están contemplando y transmitiendo nociones sobre genómica poblacional y su relación con enfermedades población-específicas.

Los/las profesionales que obtuvieron una formación extra en genética, como un curso, escuela o seminario, tienen en cuenta la etnia del paciente en mayor medida que quienes que no recibieron este tipo de formación, siendo esta diferencia estadísticamente significativa. Este dato indica que es importante profundizar, actualizar y reforzar la formación y los contenidos

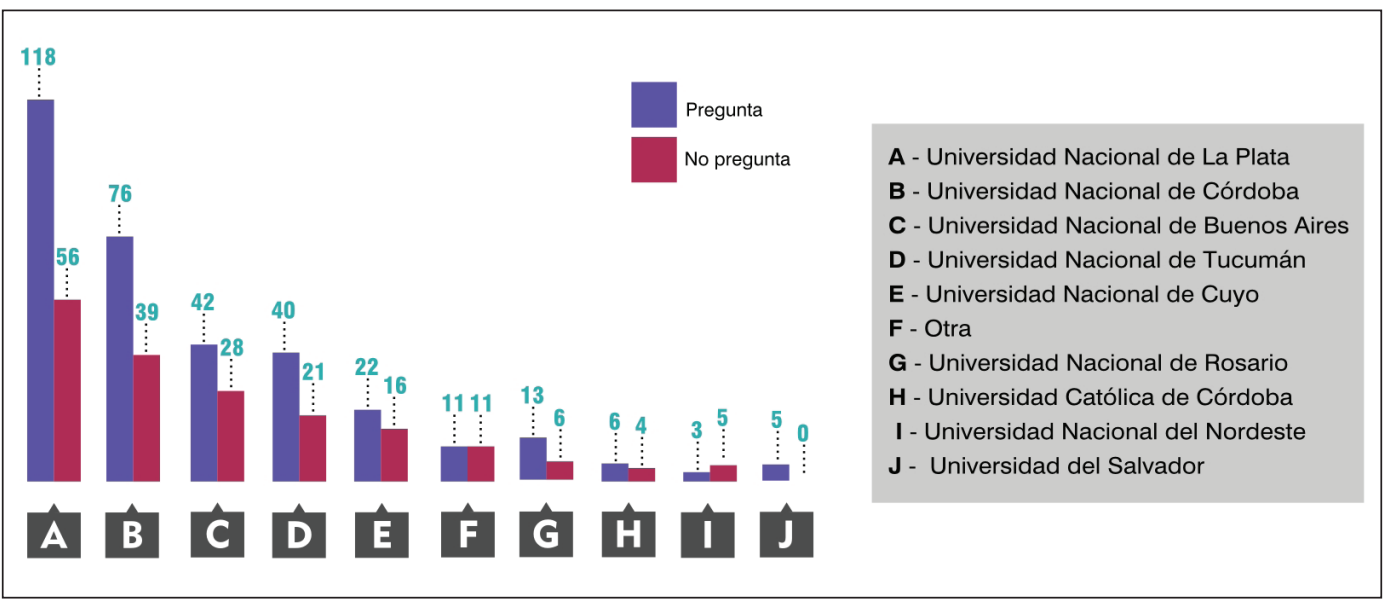

Fig. 2. ¿Pregunta por origen geográfico o étnico? según universidad de procedencia. 
sobre genómica poblacional desde los cimientos mismos de la formación durante la carrera universitaria, así como también estimularla en instancias de posgrado. Cabe resaltar que el porcentaje de encuestados/as que realizaron cursos, escuelas o seminarios sobre genética fue mucho menor $(28,9 \%)$ que el porcentaje de quienes no adquirieron este tipo de formación $(71,1 \%)$, indicando que no es un campo que médicos/as consideren prioritario para su formación.

\section{La especialidad médica}

Ciertas dolencias complejas presentan frecuencias diferenciales en poblaciones de distinto origen. La diabetes es una de ella, registra una incidencia elevada en poblaciones de origen amerindio comparado a poblaciones de origen europeo. Sobre la base de esta evidencia se definió el criterio para separar la base de datos y observar por grupos si determinados especialistas en enfermedades complejas -obesidad, diabetes y sus dolencias asociadas- tienen una consideración especial sobre la variable etnia, pero no se observaron diferencias entre las personas consultadas en la encuesta.

\section{El lugar de la medicina de precisión en Argentina}

La investigación e identificación de variantes genéticas de predisposición a enfermedades población-específicas, es un campo de notable avance en los últimos años. Sin embargo, presenta obstáculos tanto en su fase de investigación como de aplicación cuando se trata de poblaciones mestizas.

Algunos países con alto PBI, como Estados Unidos y algunas naciones europeas, destinan un gran presupuesto a la investigación, a diferencia de países con PBI más bajos como los de Latinoamérica, Asia y África. Por ejemplo, el presupuesto actual del Instituto Nacional de Salud de Estados Unidos (NIH por sus siglas en inglés) es de \$34 billones de dólares (Conte, Liu, Schnell y Omary, 2017). Este instituto estatal cuenta con una clínica para investigación genómica (ClinGen), abocada exclusivamente a arrojar luz sobre las variantes genéticas y sus conexiones con la salud y la enfermedad. El presupuesto de investigación en ese país es casi 5 veces mayor que el de Argentina: Estados Unidos destina el 2,74\% de su PBI en I+D (Investigación+Desarrollo), Argentina solo el 0,54\% (UNESCO, 2019).

Nuestro país aún no resuelve las profundas desigualdades sociales y económicas, que conllevan a disparidades en el acceso y la calidad de la atención sanitaria (Kessler, 2014). Está sujeta a vaivenes económicos y políticos que dificultan el desarrollo a largo plazo de programas de investigación que tengan impacto en la salud. Por ejemplo, no cuenta con un biobanco de referencia de su población, que permita realizar investigación biomédica de base genómica y variables asociadas. Hasta el momento, los estudios sobre la estructura genética fina han sido a pequeña escala, por lo cual los conocimientos sobre su variabilidad no son suficientes para estudiar la asociación de la ancestría genética con enfermedades de gran incidencia en nuestra población.

Esta situación representa una desventaja tanto a nivel local como internacional, se ha demostrado en varios estudios que para que la medicina de precisión personalizada sea efectiva, es necesario que se considere la diversidad genética humana de manera exhaustiva (Petrovski y Goldstein, 2016).

La falta de presupuesto, infraestructura y tradición de investigación a gran escala, que permitan caracterizar la variabilidad genética poblacional y su relación con la epidemiología de enfermedades complejas, hace que la medicina de precisión aún no sea prioridad en la agenda de médicos/as y formadores de profesionales en Argentina. A su vez, la población presenta el desafío agregado de ser mestiza: la heterogeneidad genética exige mayores esfuerzos de muestreo para una correcta caracterización genómica. Este desafío es compartido también con médicos/as, quienes deben poder distinguir entre las estrategias a aplicar en poblaciones genéticamente homogéneas y en el contexto de una población mestiza.

\section{La importancia de las variables sociales y culturales}

Las nociones socialmente construidas acerca de la raza, arraigadas durante siglos en las sociedades latinoamericanas y del mundo, tuvieron repercusiones significativas en el plano de la 
salud. Por ejemplo, en Estados Unidos hay grandes diferencias entre afroamericanos y europeos en cuanto a factores socio-económicos como la educación, los ingresos, las tasas de pobreza y las tasas de cobertura de salud (Williams, 2005). Por otro lado, Perreira y Telles (2014) observaron que, en América Latina, las personas auto-percibidas como indígenas autocalifican su salud más baja que quienes se perciben como no indígenas. En el caso de la obesidad, varios estudios sugieren que el acceso a tratamientos no es igualitario entre subgrupos específicos, como los descendientes de nativos americanos que viven en condiciones aisladas o marginales (Montenegro y Stephens, 2006), o personas de bajos ingresos (Bastos, Barros, Celeste, Paradies y Faerstein, 2004; Ortiz-Hernandez, Pérez-Salgado y Tamez-González, 2015).

Esta segregación continúa hasta el presente y sigue contribuyendo a las disparidades de salud. Atribuir las diferencias entre grupos poblacionales sólo a las diferencias en la biología subyacente es incorrecto e insuficiente, y puede ser peligroso si no son consideradas las variables socio-económicas y culturales (Cheng y Goodman, 2014; Mersha y Abebe, 2015). No incluir estas variables en los estudios de relación entre ancestría genética y riesgo a enfermedad, tanto como en la práctica médica, puede conducir a inferencias erróneas, reforzar los estereotipos raciales (Lipman y Tiedje, 2006) y profundizar las brechas de disparidad en salud entre grupos poblacionales de distintos origen.

\section{LITERATURA CITADA}

Bains, R. K., Kovacevic, M., Plaster, C. A., Tarekegn, A., Bekele, E., Bradman, N. N., y Thomas, M. G. (2013). Molecular diversity and population structure at the Cytochrome P450 3A5 gene in Africa. BMC genetics, 14(1), 34. doi:10.1186/1471-2156-14-34

Bastos, J. L., Barros, A. J., Celeste, R. K., Paradies, Y., y Faerstein, E. (2014). Age, class and race discrimination: their interactions and associations with mental health among Brazilian university students. Cadernos de Saúde Pública, 30(1), 175-186.

Baye, T. M., Wilke, R. A., y Olivier, M. (2009). Genomic and geographic distribution of private SNPs and pathways in human populations. Personalized Medicine, 6, 623-641. doi:10.2217/pme.09.54

Cheng, T. L., y Goodman, E. (2014). Race, ethnicity, and socioeconomic status in research on child health. Pediatrics, 135(1), e225-e237. doi:10.1542/peds.2014-3109

Conte, M. L., Liu, J., Schnell, S., y Omary, M. B. (2017). Globalization and changing trends of biomedical re- search output. JCI insight, 2(12), e95206. doi:10.1172/ jci.insight.95206

Kessler, G. (2014). Controversias sobre la desigualdad: Argentina, 2003-2013. Ciudad Autónoma de Buenos Aires, Argentina: Fondo de Cultura Económica.

Landry, L. G., y Rehm, H. L. (2018). Association of racial/ ethnic categories with the ability of genetic tests to detect a cause of cardiomyopathy. JAMA Cardiology, 3(4), 341-345. doi:10.1001/jamacardio.2017.5333

Landry, L. G., Ali, N., Williams, D. R., Rehm, H. L., y Bonham, V. L. (2018). Lack of diversity in genomic databases is a barrier to translating precision medicine research into practice. Health Affairs, 37(5),780-785. doi:10.1377/hlthaff.2017.1595

Lipman, T., y Tiedje, L. B. (2006). Genetic research and health disparities. The American Journal of Maternal/ Child Nursing, 31(3), 204. doi:10.1097/00005721200605000-00017

Mersha, T. B., y Abebe, T. (2015). Self-reported race/ethnicity in the age of genomic research: its potential impact on understanding health disparities. Human Genomics, 9:1. doi:10.1186/s40246-014-0023-x

Montenegro, R. A., y Stephens, C. (2006). Indigenous health in Latin America and the Caribbean. Lancet,367(9525), 1859-1869. doi:10.1016/S0140-6736(06)68808-9

Ortiz-Hernández, L., Pérez-Salgado, D., y Tamez-González, S. (2015). Socioeconomic inequality and health in Mexico. Revista Médica del Instituto Mexicano del Seguro Social, 53(3), 336-347.

Parra, F. C., Amado, R. C., Lambertucci, J. R., Rocha, J., Antunes, C. M., y Pena, S. D. (2003). Color and genomic ancestry in Brazilians. Proceedings of the National Academy of Sciences of the United States of America, 100(1), 177-182.

Perreira, K. M., y Telles, E. E. (2014). The color of health: skin color, ethnoracial classification, and discrimination in the health of Latin Americans. Social Science \& Medicine, 116, 241-250.

Petrovski, S. y Goldstein, D. B. (2016). Unequal representation of genetic variation across ancestry groups creates healthcare inequality in the application of precision medicine. Genome Biology, 17(1). doi:10.1186/s13059016-1016-y

Popejoy, A. B., Ritter, D. I., Crooks, K., Currey, E., Fullerton, S. M., .... Hindorff, L. A. (2018). The clinical imperative for inclusivity: Race, ethnicity, and ancestry (REA) in genomics. Human Mutation, 39(11), 17131720. doi:10.1002/humu. 23644

Ramos, E. y Weissman, S. M. (2018). The dawn of consumer-directed testing. American Journal of Medical Genetics, 178(1), 89-97. doi:10.1002/ajmg.c.31603

Ruiz-Linares, A., Adhikari, K., Acuña-Alonzo, V., QuintoSanchez, M., Jaramillo, C., Arias, W., .... GonzalezJosé, R. (2014). Admixture in Latin America: geographic structure, phenotypic diversity and self-perception of ancestry based on 7,342 individuals. PLoS Genetics, 10(9), e1004572. doi:10.1371/journal.pgen.1004572

Travassos, C., y D. R., Williams. (2004). The concept and measurement of race and their relationship to public health: a review focused on Brazil and the United States. Cadernos de Saúde Pública, 20(3), 660-678.

UNESCO Institute for Statistics. (2019). Gasto en investigación y desarrollo (\% del PIB). Recuperado de:https:// datos.bancomundial.org

Williams, D. R. (2005). The health of U.S. racial and ethnic populations. The journals of gerontology. Series B, Psychological sciences and social sciences, 60 (Special issue 2), 53-62. doi:10.1093/geronb/60.Special_Issue_2.S53 